\title{
CONTINUITY OF THE EXPLOSION TIME IN STOCHASTIC DIFFERENTIAL EQUATIONS
}

\author{
JULIAN FERNÁNDEZ BONDER, PABLO GROISMAN, AND JULIO D. ROSSI
}

\begin{abstract}
Stochastic ordinary differential equations may have solutions that explode in finite time. In this article we prove the continuity of the explosion time with respect to the different parameters appearing in the equation, such as the initial datum, the drift and the diffusion.
\end{abstract}

\section{INTRODUCTION}

In this paper we consider the following stochastic differential equation (SDE):

$$
d x(t)=b(x(t)) d t+\sigma(x(t)) d w(t)
$$

with $x(0)=x_{0} \in \mathbb{R}_{\geq 0}$. Here $b$ and $\sigma$ are smooth positive functions (the precise assumptions will be stated later) and $w$ is a (one-dimensional) Wiener process defined on a given complete probability space $(\Omega, \mathcal{F}, \mathbb{P})$ with a filtration $\left\{\mathcal{F}_{t}\right\}_{t \geq 0}$ satisfying the usual conditions (i.e. it is right continuous and $\mathcal{F}_{0}$ contains all $\mathbb{P}$-null sets, [8]). Equation $(P)$ is understood in the sense of Itô.

It is well known that stochastic differential equations like $(P)$ may explode in finite time. That is, trajectories may diverge to infinity as $t$ goes to some finite time $T$ that in general depends on the particular path, see Definition 3.1.

The Feller Test for explosions (see $[8,10]$ ) gives a precise description in terms of $b$ and $\sigma$ of whether explosions in finite time occur with probability zero, positive or one. For example, if $b$ and $\sigma$ behave like powers at infinity, i.e., $b(x) \sim x^{p}$ and $\sigma(x) \sim x^{q}$ as $x \rightarrow \infty$, applying the Feller test one obtains that solutions to $(P)$ explode with probability one if $p>\max \{2 q, 1\}$. We use $f(x) \sim g(x)$ to mean that there exist constants $0<c<C$ such that $c g(x) \leq f(x) \leq C g(x)$ for large enough $x$. The intuition behind this condition is that $p>2 q$ ensures that the asymptotic behavior of the solutions is governed by the drift term while $p>1$ impose the solution to grow up so fast that explodes in finite time, as happens in the deterministic case $(\sigma=0)$.

Key words and phrases. Explosion, stochastic differential equations.

AMS Subject Classification: 60H10, 34F05.

Supported by Universidad de Buenos Aires under grant X078, by ANPCyT PICT 2006-290, and CONICET (Argentina). 
Stochastic differential equations with explosions have been considered in applications, for example, in fatigue cracking (fatigue failures in solid materials) with $b$ and $\sigma$ of power type, see [14], where solutions may explode in finite time. This explosion time is generally random, depends on the particular sample path and corresponds to the time of ultimate damage or fatigue failure in the material.

For deterministic one-dimensional ODEs $(\sigma=0)$, the dependence of the explosion time $T$ with respect to the different parameters entering in the problem is very well understood, thanks to the explicit formula

$$
T=\int_{x_{0}}^{\infty} \frac{1}{b(x)} d x
$$

That is, if $T$ is finite then the solution is defined just up to time $T$ and if $T=+\infty$ the solution is globally define. In the first case the solution explodes at time $T$, while in the later case, the solution can be either bounded or unbounded.

In more general situations ( $N$-dimensional deterministic ODEs, SDEs or parabolic PDEs), where no such explicit formula is available, the situation gets a lot more complicated.

In parabolic semilinear PDEs, typically of the form $u_{t}-\Delta u=u^{p}$, for example with Dirichlet boundary conditions, the continuous dependence of the explosion time on the initial data has deserved a great deal of attention and effort. See for instance $[1,6,7,11,12]$ and also [13] for a general result on the continuity of the explosion time in a general semiflow context.

For systems of (deterministic) ODEs, or even for nonautonomous one-dimensional ODEs, there is no general result concerning the continuous dependence of the explosion time with respect to the initial data or with respect to parameters. Up to our knowledge, the only result that treats a related issue for SDEs is [5] where the authors analyze the behavior of the explosion time under small stochastic perturbations of a one-dimensional ODE.

This paper consists in an abstract result on continuity of the explosion time under structural hypotheses and, as an application of this result, we get the continuity of the explosion time in stochastic differential equations with respect to the initial datum, the drift and the diffusion.

The main idea used in the proofs is to obtain estimates for the first time where two solutions spread at a fixed distance. This idea was previously used in [4,6] and [13]. The main results on this paper can be summarized as follows:

(1) Assume $\frac{b}{\sigma}-\frac{1}{2} \sigma^{\prime}$ is nondecreasing, $x(t)$ is a solution to $(P)$ with initial datum $x_{0}$ and $x_{n}(t)$ is a solution to $(P)$ with initial datum $x_{n}$. Let $T$ and $T_{n}$ be the explosion times for $x(t)$ and $x_{n}(t)$ respectively. If $x_{n} \rightarrow x_{0}$ then $T_{n} \rightarrow T$ a.s.

(2) For additive ( $\sigma$ constant) or multiplicative ( $\sigma$ linear) noise, under adequate hypotheses, if $b_{n} \rightarrow b$ and $\sigma_{n} \rightarrow \sigma$ then $T_{n} \rightarrow T$ a.s. 
Organization of the paper. In Section 2 we prove an abstract result on convergence of explosion times for sequences of functions with explosion; in Section 3 we recall some known results on the relation between SDEs and random differential equations, namely the Doss-Sussmann theory; in Section 4 we study the dependence of $T$ with respect to the initial datum; finally in Section 5 we look at the dependence of $T$ with respect to $b$ and $\sigma$ in two relevant examples; additive and multiplicative noise.

\section{An ABstract Result}

In this section we prove a very general result on convergence of the explosion times. Let $T_{n}, T$ be real numbers and $u_{n}:\left[0, T_{n}\right) \rightarrow X, u:[0, T) \rightarrow X$ continuous functions with values on a Banach space $(X,\|\cdot\|)$, such that the following hypotheses hold:

Continuation property:

$$
\lim _{t \rightarrow T^{-}}\|u(t)\|=\infty, \quad \lim _{t \rightarrow T_{n}^{-}}\left\|u_{n}(t)\right\|=\infty .
$$

That is, we assume that both $u$ and $u_{n}$ explode in finite times, $T$ and $T_{n}$ respectively.

Continuous dependence:

For every $t<T$ it holds: $t<T_{n}$ for $n \geq n_{0}(t)$ and

$$
\lim _{n \rightarrow \infty} \sup _{s \in[0, t]}\left\|u_{n}(s)-u(s)\right\|=0 .
$$

That is, we are assuming that $u_{n}$ approaches $u$ as $n \rightarrow \infty$ at times at which $u$ is well defined and bounded.

Uniform upper explosion estimate:

There exists a nondecreasing continuous function $G:[0,+\infty) \rightarrow \mathbb{R}$, independent of $n$, such that

$$
\left\|u_{n}(t)\right\| \leq G\left(\frac{1}{T_{n}-t}\right), \quad t<T_{n} .
$$

We are assuming that we have a uniform (in $n$ ) bound on the explosion rate of the sequence $u_{n}$.

The main result of the section, is the following:

Theorem 2.1. If (H1)-(H3) hold, then

$$
\lim _{n \rightarrow \infty} T_{n}=T \text {. }
$$

We divide the proof of the theorem into two propositions.

Proposition 2.2. If (H1)-(H3) hold, then

$$
\limsup _{n \rightarrow \infty} T_{n} \leq T .
$$


Proof. It is enough to consider $n$ such that $T_{n}>T$. Set, for $t<T$

$$
e_{n}(t)=\left\|u_{n}(t)-u(t)\right\| .
$$

We have $e_{n}(0)=o(1)$. Assume that for all $t<T, e_{n}(t)<1$, then $T_{n} \leq T$ due to (H1); but this is impossible. Hence, there exists a first time $t_{n}<T$ such that $e_{n}\left(t_{n}\right)=1$. Hypotheses (H2) implies that $t_{n} \rightarrow T$ since for any subsequence $t_{n_{k}}$ satisfying $\sup t_{n_{k}}<T$ we have $1=e\left(t_{n_{k}}\right) \rightarrow 0$. Finally from (H3) we get

$$
G\left(\frac{1}{T_{n}-t_{n}}\right) \geq\left\|u_{n}\left(t_{n}\right)\right\| \geq\left\|u\left(t_{n}\right)\right\|-1 \rightarrow \infty, \quad n \rightarrow \infty .
$$

Recall that, due to $(\mathrm{H} 3), G(s) \rightarrow \infty$ implies that $s \rightarrow 0$. Consequently, $T_{n}-t_{n} \rightarrow 0$ as $n \rightarrow \infty$ and hence

$$
\limsup _{n \rightarrow \infty} T_{n} \leq \limsup _{n \rightarrow \infty} T_{n}-t_{n}+T=T,
$$

as we wanted to prove.

The lower semicontinuity is an easy consequence of continuation and continuous dependence properties. We recall the following to complete the proof of the theorem.

Proposition 2.3. If (H1) and (H2) hold, then

$$
\liminf _{n \rightarrow \infty} T_{n} \geq T \text {. }
$$

Proof. We need only consider $n$ such that $T_{n}<T$. We use the same notation as in the previous proof. By (H1), there is a first time $t_{n}<T_{n}$ such that $e_{n}\left(t_{n}\right)=1$ and (H2) implies that $t_{n} \rightarrow T$. Since $T_{n}>t_{n}$, it follows that $\liminf _{n \rightarrow \infty} T_{n} \geq T$, as we wanted to prove.

A counterexample. Let us now see that if (H3) fails we can have a sequence verifying (H1) and (H2) but with $T_{n} \nrightarrow T$. In order to see this fact it suffices to consider a one dimensional deterministic ODE. In fact, let us consider

$$
\left\{\begin{array}{l}
\dot{u}=u^{2} \\
u(0)=1
\end{array}\right.
$$

The explicit solution is

$$
u(t)=\frac{1}{1-t}, \quad(0<t<1)
$$

Now take

and consider

$$
f_{n}(x)= \begin{cases}x^{2} & 0 \leq x \leq n, \\ a_{n} x^{p_{n}}+\left(n^{2}-a_{n} n^{p_{n}}\right) & x>n,\end{cases}
$$

$$
\left\{\begin{array}{l}
\dot{u}_{n}=f_{n}\left(u_{n}\right) \\
u_{n}(0)=1
\end{array}\right.
$$


Remark that $u_{n}$ and $u$ coincide until they reach level $n$, that is,

$$
u_{n}(t)=u(t) \quad \text { for all } t \leq 1-\frac{1}{n} .
$$

Therefore $u_{n}$ is a solution of

$$
\left\{\begin{array}{l}
\dot{u}_{n}=a_{n} u_{n}^{p_{n}}+\left(n^{2}-a_{n} n^{p_{n}}\right), \\
u_{n}(1-1 / n)=n .
\end{array}\right.
$$

Assume that $p_{n}>1$, then $u_{n}$ explodes in finite time $T_{n}$ (hypothesis (H1)) and, from (2.2) it is clear that (H2) holds.

From (2.3), we obtain

$$
T_{n}=1-\frac{1}{n}+\int_{n}^{+\infty} \frac{d s}{a_{n} s^{p_{n}}+\left(n^{2}-a_{n} n^{p_{n}}\right)},
$$

that is, changing variables, $n u=s$,

$$
T_{n}=1-\frac{1}{n}+\int_{1}^{+\infty} \frac{d u}{n^{p_{n}-1} a_{n}\left(u^{p_{n}}-1\right)+n} .
$$

Let us choose

$$
a_{n}=\frac{1}{n^{p_{n}-1}},
$$

we obtain,

$$
T_{n}=1-\frac{1}{n}+\int_{1}^{+\infty} \frac{d u}{\left(u^{p_{n}}-1\right)+n} \geq 1-\frac{1}{n}+\int_{(n+1)^{1 / p_{n}}}^{+\infty} \frac{d u}{2 u^{p_{n}}} .
$$

Therefore, if we choose

we get

$$
p_{n}=1+\frac{1}{n}
$$

$$
T_{n} \geq 1-\frac{1}{n}+\frac{n}{2(n+1)^{\frac{1}{n+1}}} \rightarrow+\infty, \quad n \rightarrow \infty .
$$

Therefore

$$
\lim _{n \rightarrow \infty} T_{n}=+\infty \neq T=1 .
$$

It is clear that we can modify this example in such a way that $\left(f_{n}\right)_{n} \subset C^{\infty}$ (here they are only continuous) and moreover for any $K>1$ we can select $p_{n}, a_{n}$ in such a way that

$$
T_{n} \rightarrow K, \quad n \rightarrow \infty .
$$

The main obstruction that prevents convergence of the explosion times in this example is the fact that the sources $f_{n}$ and $f$ are far away at infinity for every $n$ in such a way that $f_{n}$ grows very slowly making the blow-up times increase (and even go to infinity) with $n$. In the one dimensional autonomous deterministic case, $\dot{u}=f(u)$ and $\dot{u}_{n}=f_{n}\left(u_{n}\right)$ with the same initial datum $u(0)=u_{n}(0)=u_{0}$, a sufficient condition to assure (H3) is $f_{n} \geq g$ with 
$\int_{u_{0}}^{\infty} \frac{d u}{g(u)}<\infty$. This fact plus pointwise convergence of $f_{n}$ to $f$ implies the convergence of the blow-up times, $T_{n} \rightarrow T$. This can be easily proved using the explicit formula for the explosion times

$$
T_{n}=\int_{u_{0}}^{+\infty} \frac{1}{f_{n}(s)} d s
$$

and the Dominated Convergence Theorem.

\section{Stochastic Differential Equations with explosions}

In this section we extend some well known results for SDEs with global solutions to the case where explosions occurs. The techniques are not new and quite standard but we include them for the sake of completeness since they are scattered in the literature.

\subsection{Existence and uniqueness.}

Definition 3.1. A strong solution up to an explosion time of equation $(P)$ is a continuous, $\mathcal{F}_{t}$ adapted process $x=\{x(t), 0 \leq t<\infty\}$ with values in $\mathbb{R} \cup\{ \pm \infty\}$ with $|x(0)|<\infty$ a.s. that verifies that for $S_{N}=\inf \{t \geq 0,|x(t)| \geq N\}$, we have, for every $N \geq 1$,

$\mathbb{P}\left[\int_{0}^{t \wedge S_{N}} b(x(s))+\sigma^{2}(x(s)) d s<\infty\right]=1, \quad \forall 0 \leq t \leq \infty \quad$ and
$\mathbb{P}\left[x\left(t \wedge S_{N}\right)=x_{0}+\int_{0}^{t} b(x(s)) \mathbf{1}\left\{s \leq S_{N}\right\}+\int_{0}^{t} \sigma(x(s)) \mathbf{1}\left\{s \leq S_{N}\right\} d w(s), \forall 0 \leq t<\infty\right]=1$,

We refer to

$$
T=\lim _{N \rightarrow \infty} S_{N}
$$

as the explosion time for $x$.

Observe that from the continuity of $x$ we have

$$
T=\inf \{t \geq 0, x(t) \notin \mathbb{R}\} \text { and } x(T)= \pm \infty \text { a.s. on }\{T<\infty\}
$$

The assumption of finiteness of $x_{0}$ gives $\mathbb{P}[T>0]=1$.

A standard truncation argument gives the existence and uniqueness of a strong solution up to an explosion time for equation $(P)$.

Theorem 3.2. Let $b$ and $\sigma$ be locally Lipschitz functions, then there exists a unique strong solution to $(P)$ up to an explosion time $T$. 
Proof. Let

$$
b_{N}(x)= \begin{cases}b(x) & \text { if }|x| \leq N \\ b(N) & \text { if } x \geq N \\ b(-N) & \text { if } x \leq-N\end{cases}
$$

and

$$
\sigma_{N}(x)= \begin{cases}\sigma(x) & \text { if }|x| \leq N \\ \sigma(N) & \text { if } x \geq N \\ \sigma(-N) & \text { if } x \leq-N .\end{cases}
$$

and consider $x_{N}$ the unique strong solution of

$$
d x_{N}=b_{N}\left(x_{N}\right) d t+\sigma_{N}\left(x_{N}\right) d w, \quad x_{N}(0)=x_{0} .
$$

Existence and uniquenes holds here since $b_{N}$ and $\sigma_{N}$ are globally Lipschitz. Let $S_{N}=$ $\inf \left\{t \geq 0,\left|x_{N}(t)\right| \geq N\right\}$. It is clear that $\left\{S_{N}\right\}_{N \geq 1}$ is monotone increasing and hence it converges to a limit $T$ that could be infinity. Moreover $x_{N+1}\left(t \wedge S_{N}\right)=x_{N}\left(t \wedge S_{N}\right)$ and so $\left\{x_{N}\right\}_{N \geq 1}$ is also convergent. Let

$$
x=\lim _{N \rightarrow \infty} x_{N} .
$$

It is easy to see that $x$ is a strong solution up to an explosion time and that $T$ is the explosion time. Following the same arguments uniqueness follows from the uniqueness for globally Lipschitz coefficients.

3.2. Doss-Sussmann theory for exploding solutions. As proved in [15] (see also [8] pages 295-297), if $\sigma$ is of class $C^{2}(\mathbb{R})$ one can get a solution to $(P)$ by means of a random differential equation. In order to do that it is preferable to consider the solution $x$ of $(P)$ as a solution of a SDE in Fisk-Stratonovich form. Assume for the moment that $b$ is globally Lipschitz and $\sigma$ has bounded first and second derivatives. Let $\beta:=b-\frac{1}{2} \sigma \sigma^{\prime}$. Then $(P)$ is equivalent to

$$
d x(t)=\beta(x(t)) d t+\sigma(x(t)) \circ d w(t) .
$$

Here we use o to denote that the equation is in Fisk-Stratonovich form.

Let us define $\phi(t, z)$ the flux associated to the ODE

$$
\dot{\phi}(t, z)=\sigma(\phi(t, z)), \quad \phi(0, z)=z .
$$

Observe that, as $\sigma$ is globally Lipschitz, $\phi(t, z)$ is globally defined. Let

$$
H(z, t):=\frac{\beta(\phi(t, z))}{\partial_{z} \phi(t, z)} .
$$

Observe that

$$
H(z, t)=\frac{\beta(\phi(t, z)) \sigma(z)}{\sigma(\phi(t, z))} .
$$


In fact,

$$
\frac{\sigma(\phi(t, z))}{\sigma(z)}=\partial_{z} \phi(t, z),
$$

since both are the unique solution to

$$
\dot{y}(t)=\sigma^{\prime}(\phi(t, z)) y(t), \quad y(0)=1 .
$$

Now, for any $\omega \in \Omega$ fixed, such that the path of the Wiener process $w(\cdot, \omega)$ is continuous, we consider $z(t)$ to be the solution of the (deterministic, non autonomous) ODE:

$$
\dot{z}(t, \omega)=H(z(t, \omega), w(t, \omega)) .
$$

This type of equations are known as random differential equations since the dependence on $\omega$ is just on the coefficients of an ODE.

The process $x(t)$ given by $x(t, \omega):=\phi(w(t, \omega), z(t, \omega))$ is a solution to $(P)$ with initial datum $x(0)=x_{0}$. See [8], pages 295-297 for the details.

If $b$ is locally Lipschitz continuous, solutions may explode. Therefore we apply the same truncation argument of the previous subsection to $\beta$ (we don't need to truncate $\sigma$ since it is already globally Lipschitz). We obtain a flux $\phi$ independent of the truncation and sequences $\beta_{N}, H_{N}, z_{N}$ and $S_{N}:=\inf \left\{t \geq 0,\left|z_{N}(t)\right| \geq N\right\}$. We define $x_{N}(t, \omega):=\phi\left(w(t, \omega), z_{N}(t, \omega)\right)$. As before, the following limits exist:

$$
x=\lim _{N \rightarrow \infty} x_{N}, \quad T=\lim _{N \rightarrow \infty} S_{N} .
$$

Since $x(t)=\phi\left(w(t, \omega), z_{N}(t, \omega)\right)$ for $t \leq S_{N}$ we get

$$
x(t)=\phi(w(t), z(t)), \quad t<T .
$$

As $\phi$ is globally defined, $x$ explodes if and only if $z$ does, and both variables explode at the same time $T$.

\section{Continuous Dependence With Respect to initial Data}

Now we combine the results of the previous sections to prove the continuity of the explosion time with respect to the initial data in stochastic differential equations. Recall that $\beta:=b-\frac{1}{2} \sigma \sigma^{\prime}$.

Theorem 4.1. Assume that $\sigma$ is $C^{2}$ with bounded first and second derivatibes, that $b$ is locally Lipschitz and that $\frac{\beta}{\sigma}=\frac{b}{\sigma}-\frac{1}{2} \sigma^{\prime}$ is nondecreasing. Let $x(t)$ and $x_{n}(t)$ be the solutions to $(P)$ with initial data $x_{0}$ and $x_{n}$ respectively and assume that $x_{n} \rightarrow x_{0}$. If $x(t)$ explodes at a random time $T$ with $\mathbb{P}(T<\infty)=1$, then $x_{n}(t)$ explodes at a random time $T_{n}$ with $\mathbb{P}\left(T_{n}<\infty\right)=1$ and

$$
T_{n} \rightarrow T \quad \text { a.s. in } \Omega \text {. }
$$


Proof. Thanks to the previous section we can think of $(P)$ as a random differential equation. To apply our general result proved in Section 2, we just need to show that (H1), (H2) and (H3) are verified for the pathwise solutions $z_{n}$ and $z$ of equation (3.6) with intitial values $x_{n}$ and $x_{0}$ respectively.

First observe that $H(z, t)$ is locally Lipschitz since the definition of $\phi(t, z)$ implies that $\partial_{z} \phi(t, z)>c>0$. Hypotheses (H1) holds since $H$ is monotone increasing with respect to the time variable and hence the existence (or not) of explosion does not depend on the initial datum. Hypotheses (H2) is a consequence of very well known results on continuous dependence with respect to initial data for nonautonomous ODEs (see for example [2] or Section 5.1 to see how to deal with the explosion). To prove (H3) we consider

$$
S:=\sup _{n \geq 1}\left\{T_{n} ; T\right\}
$$

The monotonicity of the explosion time with respect to initial data implies $\mathbb{P}(S<\infty)=1$. Now let $M, K>0$ be two large constants and define

$$
A_{K, M}:=\{\omega \in \Omega: S(\omega) \leq K \text { and }|w(t, \omega)| \leq M, \text { for } t \in[0, K+1]\} .
$$

Observe that

$$
\mathbb{P}\left(\bigcup_{K, M \geq 1} A_{K, M}\right)=1 .
$$

Now note that since $\beta / \sigma$ is nondecreasing it follows that $H(z, t)$ is nondecreasing in $t$, hence for $\omega \in A_{K, M}$, we have $H(z, w(t, \omega)) \geq H(z,-M)$ for every $z \in \mathbb{R}, 0 \leq t \leq S(\omega)$.

Let $z(t), z_{n}(t)$ be the solutions to (3.6) with initial data $x_{0}, x_{n}$ respectively. As mentioned previously, $\phi(w(t), z(t)), \phi\left(w(t), z_{n}(t)\right)$ solve $(P)$ and the explosion times of $z(t)$ and $z_{n}(t)$ are $T$ and $T_{n}$ respectively.

We have

Integrating we obtain

$$
\dot{z}_{n}(t)=H\left(z_{n}(t), w(t)\right) \geq H\left(z_{n}(t),-M\right) .
$$

$$
\int_{t}^{T_{n}} \frac{\dot{z}_{n}(t)}{H\left(z_{n}(s),-M\right)} \geq T_{n}-t
$$

and changing variables,

$$
\int_{z_{n}(t)}^{+\infty} \frac{d u}{H(u,-M)} \geq T_{n}-t
$$

Let

$$
g(\xi):=\left(\int_{\xi}^{+\infty} \frac{d u}{H(u,-M)}\right)^{-1} .
$$

Since $g$ is increasing, its inverse $G:=g^{-1}$ is also increasing and then we have

$$
z_{n}(t) \leq G\left(\frac{1}{T_{n}-t}\right) .
$$


Hence (H3) is also verified and the result follows.

From this theorem we obtain the following corollaries.

Corollary 4.2. Under the same hypotheses of Theorem 4.1, if $x_{0}$ and $x_{n}$ are random variables independent of the Brownian motion $w$ and with finite second moment such that $x_{n} \rightarrow x_{0}$ a.s. in $\Omega$, then $T_{n} \rightarrow T$ a.s. in $\Omega$.

Proof. This result follows exactly as in the proof of Theorem 4.1. Just observe that the arguments used there works for $\omega \in \Omega$ fixed, and so it is irrelevant if the initial datum is deterministic or not as far as $x_{n} \rightarrow x_{0}$ for almost every $\omega$.

Corollary 4.3. Under the same hypotheses of Theorem 4.1, if $x_{n} \rightarrow x_{0}$ in probability, then $T_{n} \rightarrow T$ in probability.

Proof. The proof of this corollary is just an application of Corollary 4.2, taking into account the following lemma which is straightforward and the proof is omitted.

Lemma 4.4. Let $x_{n}, x: \Omega \rightarrow \mathbb{R}$ be random variables. Then $x_{n} \rightarrow x$ in probability if and only if for every subsequence $x_{n_{k}}$ there exists a sub-subsequence $x_{n_{k_{j}}}$ such that $x_{n_{k_{j}}} \rightarrow x$ a.s. in $\Omega$.

Remark 4.1. It is worth noticing that in the case of additive ( $\sigma$ constant) and multiplicative ( $\sigma$ linear) noise, the hypotheses of Theorem 4.1 are verified if and only if the drift satisfies $\int_{y_{0}}^{\infty} b^{-1}(x) d x<+\infty$ for some $y_{0} \geq 0$. The explosion of the solutions with probability one can be checked by means of the Feller Test or by direct computation.

\section{Continuous Dependence With Respect to $b$ And $\sigma$}

In this section we show in the two most important examples, additive and multiplicative noise, how the abstract result of Section 2 can be applied to deal with perturbations of the drift and the diffusion.

The idea is as follows: First, one constructs $H$ and $H_{n}$ as in Section 3. Then one has to verify $(\mathrm{H} 1)-(\mathrm{H} 3)$ for $z(t)$ and $z_{n}(t)$ the solutions to

$$
\dot{z}=H(z, w(t)) \quad \text { and } \quad \dot{z}_{n}=H_{n}\left(z_{n}, w(t)\right)
$$

respectively with same initial datum $z(0)=z_{n}(0)=x_{0}$, for almost every $\omega \in \Omega$.

5.1. Additive noise: $\sigma$ constant. Assume that $\sigma$ is a positive constant We will assume that $b$ is an increasing locally Lipschitz function. In this case we have

$$
\phi(t, z)=z+\sigma t
$$

and

$$
H(z, t)=b(z+\sigma t)
$$


Therefore $H$ is increasing in both variables.

Assume that we have $\sigma_{n} \rightarrow \sigma$ and $b_{n} \rightarrow b$, uniformly on compact sets with

$$
b_{n}(x), b(x) \geq h(x), \quad \text { and } \int^{\infty} \frac{1}{h(x)} d x<\infty .
$$

Then, for $\omega \in A_{K, M}$ (given by (4.1))the solution of $\dot{z}_{n}(t)=H\left(z_{n}(t), w(t)\right.$ ) explodes in a finite time bounded by

$$
T_{n} \leq \int_{x_{0}}^{\infty} \frac{d x}{b(x-\sigma M)}<\infty
$$

The same argument is valid for $z$ and hence (H1) holds.

To prove (H2) we proceed as follows: fix $\omega \in A_{K, M}$ and for that $\omega$ let $t<T(\omega)$ and $R:=z(t, w(t))-1$. We consider globlly Lipschitz functions $\bar{H}_{n}(x, s), \bar{H}(x, s)$ that coincide with $H_{n}(x, s), H(x, s)$ respectively for $(x, s) \in[0, R] \times[0, t]$. Since $\bar{H}_{n} \rightarrow \bar{H}$ uniformly in $[0, R] \times[0, t]$ we can assume that the Lipschitz conditions holds with the same Lipschitz constant $L$ for all $H_{n}$ and $H$ in $[0, R] \times[0, t]$. Hence, given $\varepsilon>0$ we get that for $n$ large enough

$$
\begin{aligned}
\left|z_{n}(s)-z(s)\right| \leq & \int_{0}^{s}\left|H_{n}\left(z_{n}\left(s^{\prime}\right), w\left(s^{\prime}\right)\right)-H_{n}\left(z\left(s^{\prime}\right), w\left(s^{\prime}\right)\right)\right| d s^{\prime} \\
& +\int_{0}^{s}\left|H_{n}\left(z\left(s^{\prime}\right), w\left(s^{\prime}\right)\right)-H\left(z\left(s^{\prime}\right), w\left(s^{\prime}\right)\right)\right| d s^{\prime} \\
\leq & \int_{0}^{s} L\left|z_{n}\left(s^{\prime}\right)-z\left(s^{\prime}\right)\right|+\varepsilon d s^{\prime}
\end{aligned}
$$

Applying Gronwall's inequality we obtain for all $0 \leq s \leq t$

$$
\left|z_{n}(s)-z(s)\right| \leq \varepsilon t e^{L t} .
$$

This proves (H2). To check (H3) we observe that

$$
T-t=\int_{t}^{T} \frac{\dot{u}_{n}}{b_{n}\left(u_{n}+\sigma_{n} w\right)} \leq \int_{t}^{T} \frac{\dot{u}_{n}}{b_{n}\left(u_{n}-\sigma_{n} M\right)}=\int_{u_{n}(t)-\sigma_{n} M}^{\infty} \frac{d x}{b_{n}(x)} \leq \int_{u_{n}(t)-\sigma_{n} M}^{\infty} \frac{d x}{h(x)} .
$$

Hence, if we call

$$
g(x)=\int_{x}^{\infty} \frac{d y}{h(y)}
$$

we have, for large $n$,

$$
u_{n}(t) \leq g^{-1}(T-t)+2 \sigma M=: G\left(\frac{1}{T-t}\right) .
$$

So we can apply Theorem 2.1 to obtain the following theorem. 
Theorem 5.1. Let $\sigma_{n}, \sigma$ be positive constants such that $\sigma_{n} \rightarrow \sigma$. Assume $b$ is an increasing locally Lipschitz function and that $b_{n} \rightarrow b$ uniformly on compact sets. Assume also that there exists a function $h$ such that

$$
b_{n}(x), b(x) \geq h(x), \quad \text { with } \int^{\infty} \frac{1}{h(x)} d x<\infty .
$$

Consider $x_{n}$ and $x$ the solutions to $(P)$ with $\left(b_{n}, \sigma_{n}\right),(b, \sigma)$ up to the explosion times $T_{n}$, $T$ respectively. Then with probability one

$$
T<\infty, \quad T_{n}<\infty \text { for all } n \text { and } T_{n} \rightarrow T \text {. }
$$

5.2. Multiplicative noise: $\sigma$ linear. Assume that $\sigma(x)=a x$ with $a>0$. Recall that $\phi$ is the solution of

$$
\left\{\begin{array}{l}
\dot{\phi}=\sigma(\phi)=a \phi, \\
\phi(0, z)=z .
\end{array}\right.
$$

Therefore in this case we have $\phi(t, z)=z e^{a t}$ and hence

$$
H(z, t)=\frac{\beta\left(z e^{a t}\right)}{e^{a t}},
$$

with $\beta(x)=b(x)-\frac{1}{2} a^{2} x$.

Assume that we have $a_{n} \rightarrow a$ and $b_{n}, b$ are in the same conditions of the previous subsection. Now using the uniform convergence of $\beta_{n}$ to $\beta$ on compact sets, (H1) and (H2) hold for $\omega \in A_{K, M}$. The proof is a mimic of the one in the previous section.

Now we look for (H3). As before, we impose that

$$
b_{n}(x), b(x) \geq h(x), \quad \text { with } \int_{y_{0}}^{\infty} \frac{1}{h(x)} d x<\infty
$$

for some $y_{0} \geq 0$. Then, the same hold for $\beta_{n}, \beta$ with $h_{a}(x):=h(x)-\frac{1}{2} a^{2} x$ and proceeding as before, we obtain, for $n$ large enough,

$$
\begin{aligned}
T-t & =\int_{t}^{T} \frac{\dot{u}_{n} e^{a_{n} w}}{\beta_{n}\left(u_{n} e^{a_{n} w}\right)} \leq \int_{t}^{T} \frac{\dot{u}_{n} e^{a_{n} M}}{\beta_{n}\left(u_{n} e^{-a_{n} M}\right)} \\
& =e^{2 a_{n} M} \int_{u_{n}(t) e^{-a_{n} M}}^{\infty} \frac{d x}{\beta_{n}(x)} \leq e^{2 a_{n} M} \int_{u_{n}(t) e^{-a_{n} M}}^{\infty} \frac{d x}{h_{a}(x)} \\
& \leq e^{4 a M} \int_{u_{n}(t) e^{-\frac{a}{2} M}}^{\infty} \frac{d x}{h_{a}(x)}
\end{aligned}
$$

Hence, if we call

$$
g(x)=\int_{x}^{\infty} \frac{d x}{h_{a}(x)}
$$


we have

$$
u_{n}(t) \leq e^{\frac{a}{2} M} g^{-1}\left(\frac{T-t}{e^{4 a M}}\right)=: G\left(\frac{1}{T-t}\right)
$$

We have proved

Theorem 5.2. Let $a_{n}$, a be positive constants such that $a_{n} \rightarrow a$. Assume $b_{n}, b$ are in the conditions of Theorem 5.1 and let $\sigma_{n}(x)=a_{n} x, \sigma(x)=a x$. Consider $x_{n}$ and $x$ the solutions to $(P)$ with $\left(b_{n}, \sigma_{n}\right),(b, \sigma)$ up to the explosion times $T_{n}, T$ respectively. Then with probability one

$$
T<\infty, \quad T_{n}<\infty \text { for all } n \text { and } T_{n} \rightarrow T \text {. }
$$

Example. Both for additive and multiplicative noise, just consider $b_{n}(s)=\alpha_{n} s^{p_{n}}, b(s)=$ $\alpha s^{p}$, with $\alpha_{n} \rightarrow \alpha>0$, and $p_{n} \rightarrow p>1$.

\section{ACKNOWLEDGMENTS}

We want to thank M. Sued for several interesting discussions.

\section{REFERENCES}

[1] P. Baras and L. Cohen. Complete blow-up after $T_{\max }$ for the solution of a semilinear heat equation. $J$. Funct. Anal., 71(1):142-174, 1987.

[2] E.A. Coddington and N. Levinson Theory of ordinary differential equations. McGraw-Hill Book Company, Inc., New York-Toronto-London, 1955. xii+429 pp.

[3] V. A. Galaktionov and J. L. Vázquez. The problem of blow-up in nonlinear parabolic equations. Discrete Contin. Dyn. Syst., 8(2):399-433, 2002. Current developments in partial differential equations (Temuco, 1999).

[4] P. Groisman and J. D. Rossi. Dependence of the blow-up time with respect to parameters and numerical approximations for a parabolic problem. Asymptot. Anal., 37(1):79-91, 2004.

[5] P. Groisman and J. D. Rossi. The explosion time in stochastic differential equations with small diffusion. Electron. J. Diff. Eqns., Vol. 2007(2007), No. 140, pp. 1-9.

[6] P. Groisman, J. D. Rossi and H. Zaag. On the dependence of the blow-up time with respect to the initial data in a semilinear parabolic problem. Comm. Partial Differential Equations, 28(3-4):737-744, 2003.

[7] M. A. Herrero and J. J. L. Velázquez. Generic behaviour of one-dimensional blow up patterns. Ann. Scuola Norm. Sup. Pisa Cl. Sci. (4), 19(3):381-450, 1992.

[8] I. Karatzas and S.E. Shreve. Brownian motion and stochastic calculus, volume 113 of Graduate Texts in Mathematics. Springer-Verlag, New York, second edition, 1991.

[9] P.E Kloeden and E. Platen. Numerical solution of stochastic differential equations, volume 23 of Applications of Mathematics (New York), Springer-Verlag, 1992,

[10] H. P. McKean, Jr. Stochastic integrals. Probability and Mathematical Statistics, No. 5. Academic Press, New York, 1969.

[11] F. Merle. Solution of a nonlinear heat equation with arbitrarily given blow-up points. Comm. Pure Appl. Math., 45(3):263-300, 1992.

[12] P. Quittner. Continuity of the blow-up time and a priori bounds for solutions in superlinear parabolic problems. Houston J. Math., 29(3):757-799 (electronic), 2003. 
[13] J. D. Rossi and P. Souplet. Coexistence of simultaneous and nonsimultaneous blow-up in a semilinear parabolic system. Differential Integral Equations, 18(4):405-418, 2005.

[14] K. Sobczyk and B. F. Spencer, Jr. Random fatigue. Academic Press Inc., Boston, MA, 1992. From data to theory.

[15] H. J. Sussmann. On the gap between deterministic and stochastic ordinary differential equations. Ann. Probability, 6(1):19-41, 1978.

J. Fernández Bonder

Departamento de Matemática, FCeyn, Universidad de Buenos Aires, Pabellón I, Ciudad Universitaria (1428), Buenos Aires, Argentina.

E-mail address: jfbonder@dm.uba.ar

Personal web-page: http://mate.dm.uba.ar/ jfbonder

P. Groisman

Departamento de Matemática, FCeyn, Universidad de Buenos Aires, Pabellón I, Ciudad Universitaria (1428), Buenos Aires, Argentina.

E-mail address: pgroisma@dm.uba.ar

Personal web-page: http://mate.dm.uba.ar/ pgroisma

Julio D. Rossi

IMDEA MATEMATICAS,

C-IX, Campus UAM,

MADRID, SPAIN

On leave from Departamento de Matematica,

FCEYN UBA (1428)

Buenos Aires, Argentina.

E-mail address: jrossi@dm.uba.ar

Personal web-page: http://mate.dm.uba.ar/ jrossi 